Boise State University

ScholarWorks

$3-27-2007$

Frequency-Dependent Attenuation Analysis of Ground-Penetrating Radar Data

John H. Bradford

Boise State University 


\title{
Frequency-dependent attenuation analysis of ground-penetrating radar data
}

\author{
John H. Bradford ${ }^{1}$
}

\begin{abstract}
In the early 1990s, it was established empirically that, in many materials, ground-penetrating radar (GPR) attenuation is approximately linear with frequency over the bandwidth of a typical pulse. Further, a frequency-independent $Q^{*}$ parameter characterizes the slope of the band-limited attenuation versus frequency curve. Here, I derive the band-limited $Q^{*}$ function from a first-order Taylor expansion of the attenuation coefficient. This approach provides a basis for computing $Q^{*}$ from any arbitrary dielectric permittivity model. For Cole-Cole relaxation, I find good correlation between the first-order $Q^{*}$ approximation and $Q^{*}$ computed from linear fits to the attenuation coefficient curve over two-octave bands. The correlation holds over the primary relaxation frequency. For some materials, this relaxation occurs between 10 and $200 \mathrm{MHz}$, a typical frequency range for many GPR applications. Frequency-dependent losses caused by scattering and by the commonly overlooked problem of frequencydependent reflection make it difficult or impossible to measure $Q^{*}$ from reflection data without a priori understanding of the materials. Despite these complications, frequency-dependent attenuation analysis of reflection data can provide valuable subsurface information. At two field sites, I find well-defined frequency-dependent attenuation anomalies associated with nonaqueous-phase liquid contaminants.
\end{abstract}

\section{INTRODUCTION}

Propagating electromagnetic waves are subject to frequencydependent attenuation that depends on the effective conductivity. The effective conductivity is a function of the real component of the electric conductivity and the complex component of the dielectric permittivity. Turner and Siggins (1994) show empirically that, similar to the constant $Q$-factor often assumed in compressional-wave propagation, a constant $Q^{*}$ parameter can approximate the frequency-dependent component of ground-penetrating radar (GPR) attenuation in many materials commonly encountered in GPR investigations.

To extract $Q^{*}$ from field data, it is necessary to analyze the spectral variation of the GPR signal through time, which is the basis of timefrequency $(t-f)$ analysis (Morlet et al., 1982a, 1982b; Chakraborty and Okaya, 1995; Li and Ulrych, 1996; Tobbock et al., 1996; Bradford, 1999; Irving and Knight, 2003). With an accurate measure of the $t-f$ distribution, a number of methods are available to measure the frequency-dependent attenuation, including spectral ratios (Bradford, 1999) and the frequency-shift method (Liu et al., 1998).

Material property characterization using frequency-independent attenuation analysis is relatively common in crosswell studies (Grandjean et al., 2000; Lane et al., 2000; Peterson, 2001; Zhou and Liu, 2001; Chang et al., 2002; Day-Lewis et al., 2002; Day-Lewis et al., 2003; Goldstein et al., 2003). However, development and evaluation of frequency-dependent attenuation analysis for surface-based reflection data remain limited. Bano (2004) reviews dielectric mixing formulas and discusses frequency-dependent attenuation for materials following power-law behavior for complex dielectric permittivity. Turner (1994) and Irving and Knight (2003) describe methods for estimating $Q^{*}$ and applying a deconvolution filter to GPR data to compensate for frequency-dependent losses. Other studies suggest the potential of using reflection-attenuation analysis for direct material property characterization (Liu and Quan, 1997; Bradford, 1999; Cai and McMechan, 1999), but further work is needed. It is important to recognize that scattering can result in frequency-dependent attenuation (Morlet et al., 1982a) that has the same form as intrinsic attenuation and that the attenuation measured in field data is a combined effect of scattering and intrinsic attenuation.

Here, I derive the general function for $Q^{*}$ from the first-order Taylor expansion of the attenuation coefficient. This function provides a basis for computing $Q^{*}$ from any dielectric permittivity model. As an example, I show the relationship between the first-order $Q^{*}$ approximation and the parameters that describe Cole-Cole relaxation (Cole and Cole, 1941). Next, I review $t-f$ methods for measuring $Q^{*}$ from

Presented at the Symposium for the Application of Geophysics to Environmental and Engineering Problems, 2006. Manuscript received by the Editor July 28, 2006; revised manuscript received November 14, 2006; published online March 27, 2007.

${ }^{1}$ Boise State University, Center for Geophysical Investigation of the Shallow Subsurface, Boise, Idaho. E-mail: johnb@cgiss.boisestate.edu. (C) 2007 Society of Exploration Geophysicists. All rights reserved. 
field data and then demonstrate that frequency-dependent reflection can significantly distort the spectrum and mask the intrinsic $Q^{*}$ measurement. Finally, I show that despite a number of recognized complications, frequency-dependent attenuation analysis can be a strong indicator of variation in bulk subsurface dispersion properties. At two field sites, I demonstrate significant anomalies associated with both dense and light nonaqueous-phase liquid contaminants.

\section{METHODOLOGY}

\section{Relating $Q^{*}$ to the dielectric relaxation response}

For plane waves, the monochromatic propagating electric field is given by

$$
\widetilde{E}(\mathbf{r}, t)=\widetilde{E}_{0} e^{-i(\tilde{k} \hat{r}-\omega t)}
$$

where the complex wavenumber is given by

$$
\tilde{k}=\beta-i \alpha
$$

in which $\alpha$ and $\beta$ are defined as

$$
\alpha=\omega\left[\frac{\varepsilon \mu}{2}\left(\sqrt{1+\left(\frac{\sigma}{\varepsilon \omega}\right)^{2}}-1\right)\right]^{\frac{1}{2}}
$$

and

$$
\beta=\omega\left[\frac{\varepsilon \mu}{2}\left(\sqrt{1+\left(\frac{\sigma}{\varepsilon \omega}\right)^{2}}+1\right)\right]^{\frac{1}{2}}
$$

where $\omega$ is the angular frequency, $\varepsilon$ is the dielectric permittivity, $\sigma$ is the electric conductivity, and $\mu$ is the magnetic permeability. By equation 1 , the imaginary part of the wavenumber gives the attenuation, and the real part of the wavenumber gives the phase velocity by the relationship $v_{\mathrm{ph}}=\omega / \beta$.

In general, $\varepsilon, \mu$, and $\sigma$ are also complex quantities, and equations 3 and 4 can be given in terms of real effective permittivity $\varepsilon_{\mathrm{e}}$ and real effective conductivity $\sigma_{\mathrm{e}}$, where

and

$$
\varepsilon_{\mathrm{e}}=\varepsilon^{\prime}-\frac{\sigma^{\prime \prime}}{\omega}
$$

$$
\sigma_{\mathrm{e}}=\sigma^{\prime}+\varepsilon^{\prime \prime} \omega
$$

For radar, it is typically taken that $\sigma^{\prime}$ is the DC conductivity $\left(\sigma^{\prime}\right.$ $\left.=\sigma_{\mathrm{DC}}\right)$ and $\sigma^{\prime \prime}=0$. In the low-loss approximation, $\sigma_{\mathrm{e}} \ll \varepsilon_{\mathrm{e}} \omega$. By using this approximation and the two-term binomial expansion, equation 3 reduces to (Griffiths, 1989)

$$
\alpha=\frac{\sigma_{e}}{2} \sqrt{\frac{\mu_{0}}{\varepsilon_{e}}}
$$

Equation 7 is quite a good approximation even at the dispersive limit $\left(\sigma_{e}=\varepsilon_{e} \omega\right)$, where the error in the binomial approximation is only $6 \%$. Thus, the approximation is reasonable over the range of conditions under which GPR operates effectively. If the permittivity is real and independent of frequency, the attenuation is also frequency independent. The same approximation and assumptions show that velocity is frequency independent; therefore, there is no dispersion. However, the dielectric permittivity of real earth materials is complex. By equations 6 and 7, the attenuation is frequency dependent and given by

$$
\alpha \approx \frac{\left[\sigma_{\mathrm{DC}}+\varepsilon^{\prime \prime}(\omega) \omega\right]}{2} \sqrt{\frac{\mu_{0}}{\varepsilon^{\prime}(\omega)}} .
$$

In the Turner and Siggins (1994) approximation, the slope of the attenuation curve is linear and characterized with a constant $Q^{*}$ parameter that gives the attenuation coefficient as

$$
\alpha \approx \alpha_{0}+\frac{\sqrt{\mu_{0} \varepsilon_{\omega_{0}}^{\prime}}}{2 Q^{*}}\left(\omega-\omega_{0}\right)
$$

where $\omega_{0}$ is the reference frequency and $\alpha_{0}$ is the value of $\alpha$ at $\omega_{0}$. By taking the first-order Taylor expansion of equation 8 about some center frequency $\omega_{0}$, I derive the following linear approximation to the attenuation coefficient at reference frequency $\omega_{0}$ :

$$
\alpha=\alpha_{\omega_{0}}+\frac{1}{2} \sqrt{\frac{\mu_{0}}{\varepsilon_{\omega_{0}}^{\prime}}}\left(\varepsilon_{\omega_{0}}^{\prime \prime}+\omega_{0} \frac{\delta \varepsilon^{\prime \prime}}{\delta \omega}-\frac{1}{2 \varepsilon_{\omega_{0}}^{\prime}} \frac{\delta \varepsilon^{\prime}}{\delta \omega}\right)\left(\omega-\omega_{0}\right) .
$$

Note that the DC conductivity term is now included in $\alpha_{\omega_{0}}$. Noting that equation 10 has the same form as the Turner and Siggins approximation (equation 9), then combining equations 9 and 10 , I find $Q^{*}$ in terms of the complex permittivity:

$$
\frac{1}{Q^{*}}=\frac{1}{\varepsilon_{\omega_{0}}^{\prime}}\left(\varepsilon_{\omega_{0}}^{\prime \prime}+\omega_{0} \frac{\delta \varepsilon^{\prime \prime}}{\delta \omega}-\frac{1}{2 \varepsilon_{\omega_{0}}^{\prime}} \frac{\delta \varepsilon^{\prime}}{\delta \omega}\right)
$$

Equation 11 is the general form for the band-limited constant $Q^{*}$ approximation and requires no particular form for the complex dielectric permittivity. Equation 11 is appropriate for computing $Q^{*}$ from any differentiable form of the complex permittivity such as a superposition of Debye models that sometimes provides a good fit to earth materials (Hoekstra and Delaney, 1974).

As an example, consider the Cole-Cole model (Cole and Cole, 1941). A number of authors have used that model to describe the high-frequency dielectric response of a variety of earth materials (Hoekstra and Delaney, 1974; Taherian et al., 1990; Olhoeft and Capron, 1993) and to model GPR data (Roberts and Daniels, 1992; Powers and Olhoeft, 1996; Zeng et al., 2000). The Cole-Cole model gives the complex dielectric permittivity as

$$
\varepsilon^{\prime}-i \varepsilon^{\prime \prime}=\varepsilon_{\infty}+\frac{\varepsilon_{\mathrm{DC}}-\varepsilon_{\infty}}{1+(i \omega \tau)^{\gamma}},
$$

where $\tau$ is the primary relaxation time and the fit parameter $\gamma$ allows for the superposition of multiple relaxation mechanisms that may be observed in heterogeneous materials. Figure 1 shows real permittivity and attenuation coefficient curves for the representative set of Cole-Cole parameters given in Table 1 . These curves demonstrate that for some materials conducive to GPR investigation, measurable dispersion will occur from 50-100 MHz.

With the Cole-Cole relationship (equation 12), I can compute the derivatives in equation 11 (Appendix A), which gives $Q^{*}$ as a function of the reference frequency $\omega_{0}$. Comparing this relationship to the Turner and Siggins (1994) constant $Q^{*}$ approximation (measured by using piecewise linear fits to the attenuation coefficient curves, computed from equation 3 , over two-octave bands), I find the bandlimited linear fit correlates well with the first-order $Q^{*}$ approximation (Figure 2). 


\section{Measuring $Q^{*}$ from reflection data: Defining the dispersion parameter $D$}

The inherent nonuniqueness between reflectivity and the bulk attenuation $\alpha_{\omega_{0}}$ means that measuring $\alpha_{\omega_{0}}$ from surface GPR data is difficult or impossible. Understanding the full attenuation response requires additional low-frequency resistivity measurements. However, by measuring the shift in the signal spectrum through time using time-frequency analysis, it is possible to isolate the frequencydependent component of attenuation.

As the signal propagates, frequency-dependent attenuation causes the peak of the amplitude spectrum to shift. This spectral shift can be used to measure $Q^{*}$ (Quan and Harris, 1997). The source waveform of some commercially available pulsed GPR systems is similar to a Ricker wavelet. After propagating through a material for some time $t$, the amplitude spectrum of a Ricker wavelet is given by

$$
S_{t}=\sqrt{2} \frac{\omega^{2}}{\omega_{0}} \exp \left[-\frac{\omega^{2}}{\omega_{0}^{2}}-\frac{\alpha(\omega)}{\sqrt{\varepsilon_{\omega_{0}}^{\prime} \mu_{0}}} t\right]
$$
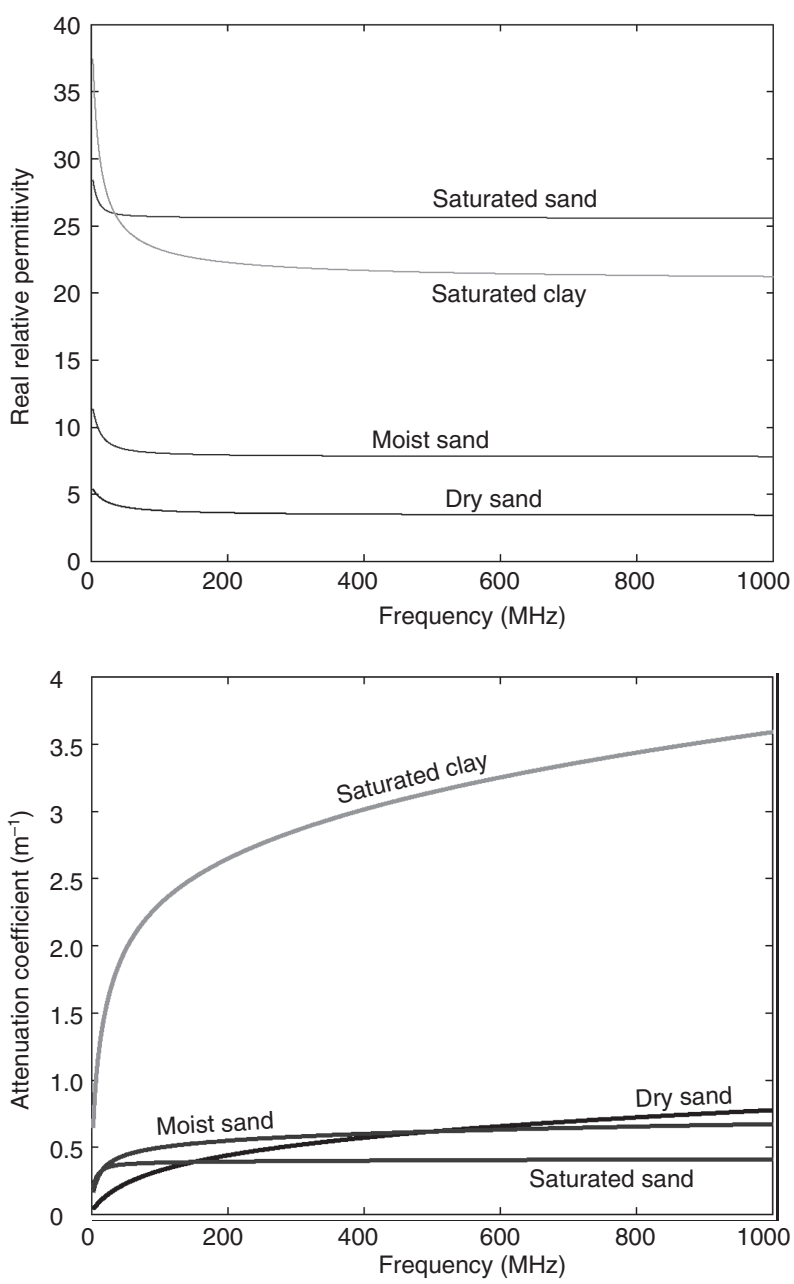

Figure 1. Frequency dependence of the real part of the dielectric permittivity and the attenuation coefficient (imaginary part of the wavenumber). The attenuation is dominated by relaxation mechanisms represented by the imaginary part of the wavenumber. where $\omega_{0}$ is the maximum of the source spectrum. By setting the derivative of equation 13 equal to zero and using equation 9 , the shift in the peak frequency of the spectrum is related to $Q^{*}$ by

$$
\frac{1}{Q^{*}}=\frac{4}{t} \frac{\left(\omega_{0}^{2}-\omega_{t}^{2}\right)}{\omega_{0}^{2} \omega_{t}},
$$

where $\omega_{t}$ is the spectral maximum at time $t$. Quan and Harris (1997) derive similar expressions for the centroid frequency shift for Gaussian and boxcar spectra. To extract $Q^{*}$ from field data, it is necessary to estimate the signal spectrum at horizons bounding the target interval. From equation 14, the spectrum of any event may be used as the reference spectrum to estimate the effective interval $Q^{*}$ for any successive event. Alternatively, the cumulative or effective $Q^{*}$ at any time $t$ can be measured if the source spectrum is known; then the interval $Q^{*}$ values can be estimated by using the approach described by Bradford (1999). Often, however, measuring the source spectrum from field data is difficult because of noise, interference between the direct airwave and direct ground wave, variations in antenna coupling, and spectral distortion caused by the low-frequency transient or wow that is observed at early times.

Table 1. Cole-Cole parameters for dielectric permittivity and magnetic permeability. The values for the three sands are given by Powers and Olhoeft (1996), and the value for clay is given by Olhoeft and Capron (1994).

\begin{tabular}{lrrrrc} 
Layer & $\varepsilon_{\mathrm{DC}} / \varepsilon_{0}$ & $\varepsilon_{\infty} / \varepsilon_{0}$ & $\begin{array}{c}\tau \\
(\mathrm{ns})\end{array}$ & $\gamma$ & $\begin{array}{c}\sigma_{\mathrm{DC}} \\
(\mathrm{mS} / \mathrm{m})\end{array}$ \\
\hline Dry sand & 5.7 & 3.4 & 8.0 & 0.70 & 0.45 \\
Moist sand & 8.9 & 5.6 & 11.0 & 0.75 & 2.00 \\
Wet sand & 29.0 & 25.6 & 22.2 & 0.88 & 6.06 \\
Clay (30\% water) & 43.0 & 20.7 & 18.3 & 0.66 & 42.5 \\
\hline
\end{tabular}

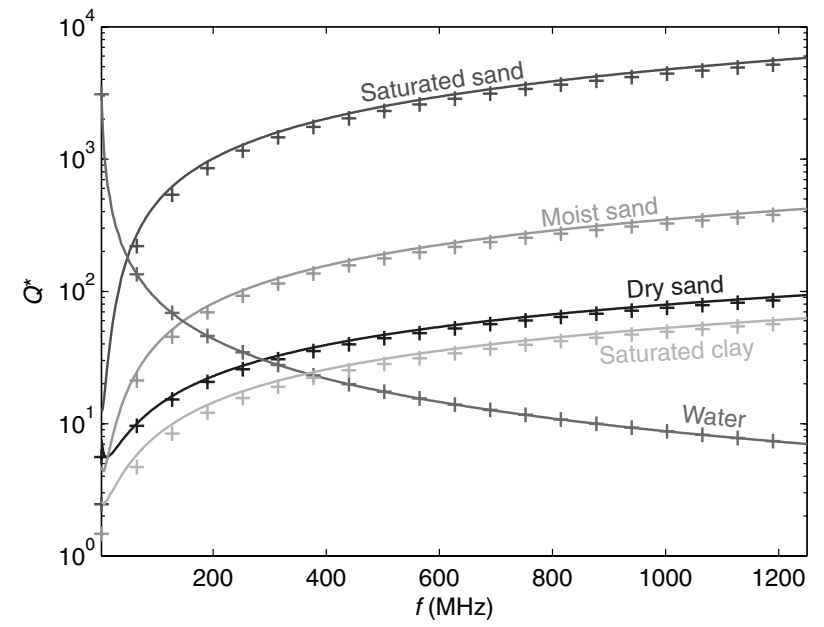

Figure 2. Lines show $Q^{*}$ as a function of frequency through the use of equation 11 and the material properties given in Table 1. Also plotted (+ signs) is $Q^{*}$ as a function of band-center frequency computed in a piecewise linear sense by fitting a line over two-octave bandwidths to equation 3 calculated with the complex permittivity given by the Cole-Cole equations. The two methods are in good agreement over the GPR frequency range. 
For attenuation analysis, I normally interpret significant horizons within a given section manually and then identify the local maximum of the envelope function within a time gate bounding the horizon. The reflection spectrum is taken from the $t$ - $f$ distribution at the
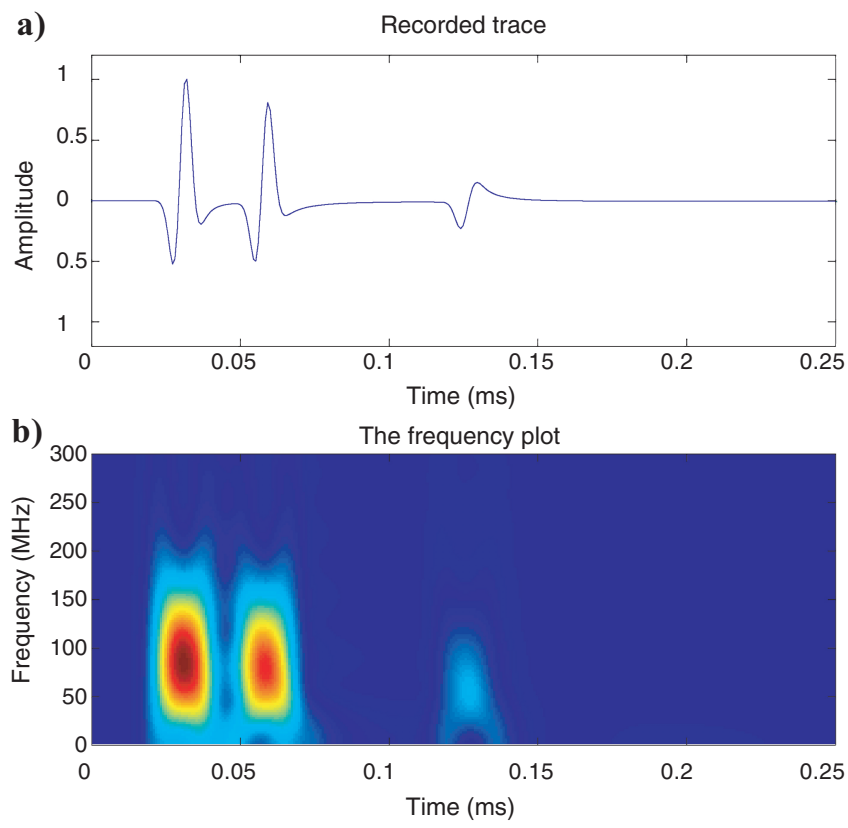

Figure 3. (a) Synthetic trace with a 100-MHz source wavelet and (b) relative-amplitude time-frequency distribution computed by using the STFT. In this model, the peak frequency decays from 85 to $56 \mathrm{MHz}$ between the first and third reflections.
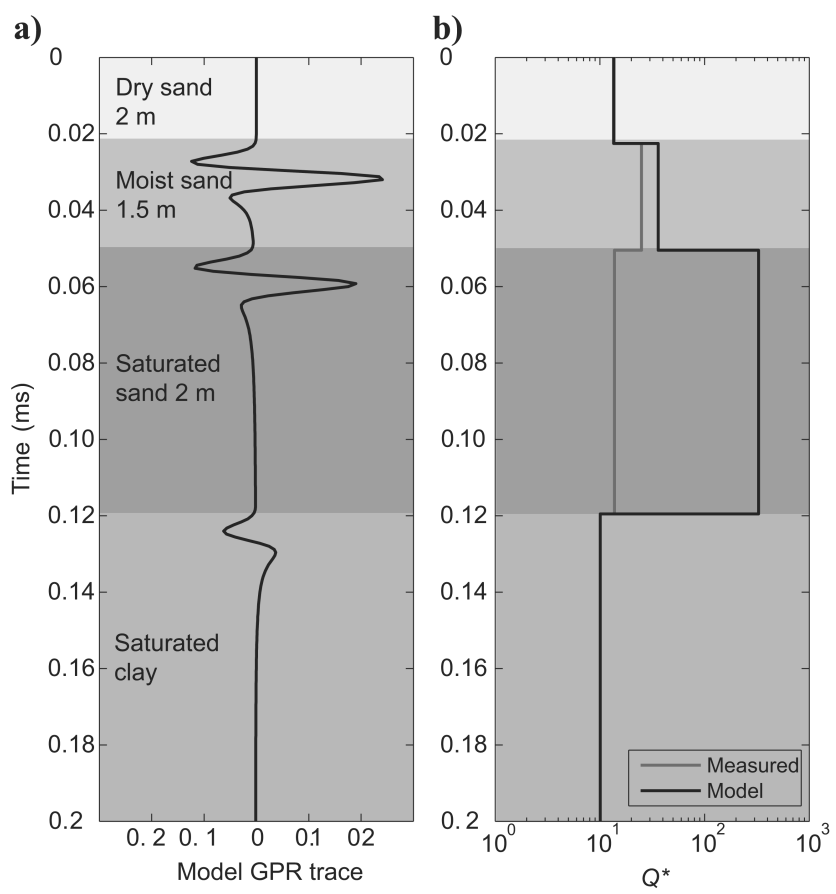

Figure 4. Model GPR data with the parameters listed in Table 1. The apparent $Q^{*}$ value diverges significantly from the actual value, particularly in the saturated sand layer. This divergence stems from spectral distortion caused by frequency-dependent reflection. peak of the envelope function for a given horizon. This approach may be targeted to a specific interval or done for all horizons within the radar section. A number of methods are available to compute the $t-f$ spectrum of the data, e.g., the short-time Fourier transform (STFT, Figure 3) or wavelet decompositions such as that described by Bradford and Wu (2007) or the S-transform as described by Irving and Knight (2003). Alternatively, if the signal-to-noise ratio $(\mathrm{S} / \mathrm{N})$ is high, the instantaneous frequency at the peak of the envelope function provides a reliable estimate of the average frequency of the reflection (Robertson and Nagomi, 1982).

One of the commonly recognized problems with $Q^{*}$ analysis is that a number of factors can alter the reflected signal spectrum that are unrelated to the intrinsic attenuation (Olhoeft and Capron, 1994). For example, scattering can result in a dispersion relationship similar to intrinsic attenuation (Morlet et al., 1982a). Therefore, scattering losses cannot be differentiated from intrinsic attenuation without a thorough description of the geometry of scatters. Additionally, although it is often overlooked, frequency-dependent reflection coefficients can significantly alter the spectrum and lead to bias in the $Q^{*}$ estimate.

As an example, by using the Cole-Cole parameters given in Table 1, I constructed a simple layered subsurface model of an unconfined aquifer in sandy sediments with a clay aquitard at the base (Figure 4). I then used a ray-based modeling code to compute the response to the model for a $100-\mathrm{MHz}$ source wavelet. The code uses the complex wavenumber in a frequency-domain computation to estimate wavelet distortion and traveltime along a given raypath (Bradford, 1999).

Comparing the interval $Q^{*}$ measured from the model data with $Q^{*}$ computed from the attenuation coefficient curves (Figure 4) makes it clear that the measured $Q^{*}$ differs dramatically from the intrinsic $Q^{*}$ value. This divergence arises entirely from changes in the dispersion curve across layer boundaries; these changes lead to strong frequency dependence in the reflection coefficients below $200 \mathrm{MHz}$ (Figure $5)$. This effect is most obvious in the reflection from the boundary between water-saturated sand and clay. At this interface, the reflection coefficient at low frequencies is larger than at high frequencies. This

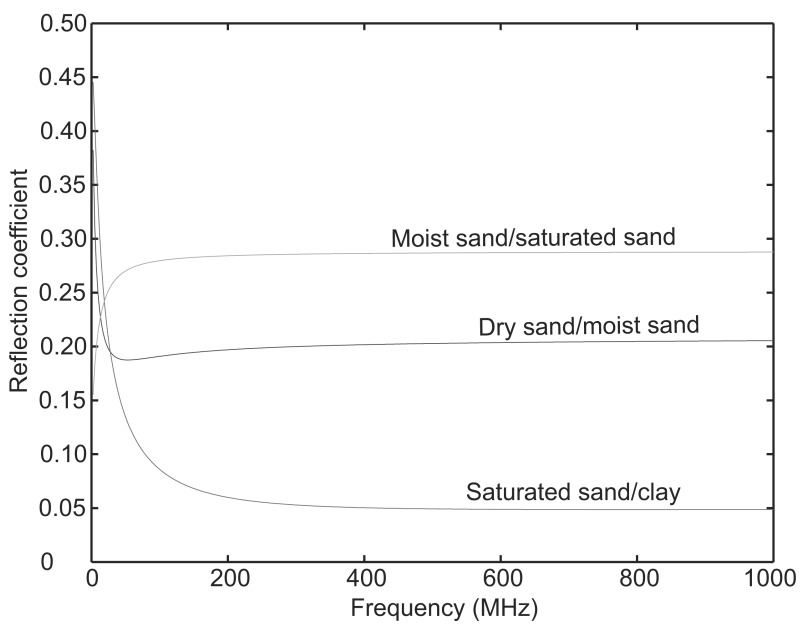

Figure 5. For the model shown in Figure 4, the reflection coefficients have strong frequency dependence below $200 \mathrm{MHz}$, particularly for the clay reflection. Spectral distortion caused by frequency-dependent reflectivity can mask the effect of intrinsic attenuation. 
difference causes a spectral shift toward lower frequencies, which makes the $Q^{*}$ value measured from the data much lower than the actual value.

Recognizing that the measured spectrum is altered by a number of mechanisms, I define the dispersion parameter $D$ as

$$
D=\frac{1}{Q_{\mathrm{app}}^{*}},
$$

where $Q_{\text {app }}^{*}$ now includes all frequency-dependent attenuation mechanisms. I use this term because velocity dispersion and frequency-dependent attenuation are linked inherently through the Kramers-Kronig relationships, which are required to meet the causality condition (Jackson, 1999). Use of the $D$ nomenclature avoids the implication of a measurement of intrinsic attenuation that defines the $Q^{*}$ parameter. $D$ is large for strong dispersion and small for weakly dispersive media.

\section{FIELD STUDIES}

Clearly the subsurface characteristics that lead to the measured $D$ value are complex; in general, it may be impossible to isolate any particular contribution to the observed spectral changes. Although it may be impossible to differentiate changes in dispersion caused by intrinsic attenuation from dispersion that results from frequencydependent reflection or other effects, the measurement can be used to look for spectral anomalies in the subsurface related to target materials.

\section{Measuring the relaxation response of freshwater}

Figure 6 shows the result of measuring $D$ for water from a 200MHz field data set acquired on the North Slope of Alaska (Bradford et al., 2005). These data were acquired over an approximately 2$\mathrm{m}$-deep pond by placing the antennas in the bottom of a small rubber boat and then pulling the boat across the pond.

The processing flow consists of band-pass filtering (12-25-400$800-\mathrm{MHz}$ passband), a $t^{2}$ gain function, and background removal (average trace subtraction method). At the time of acquisition, the pond was flooded and about $10 \mathrm{~cm}$ of water was standing on the banks. Additionally, the bank material was peat and had a dielectric permittivity very close to that of the pond water, $\varepsilon_{W} / \varepsilon_{0} \approx 87$ at $0{ }^{\circ} \mathrm{C}$. Given this observation, I assume that antenna loading does not vary across the length of the profile and that the source waveform is constant. The shallowest reflection on the bank at $34 \mathrm{~ns}$ is from the top of the permafrost table at $0.5 \mathrm{~m}$ depth. This high-amplitude, low-noise event serves as an appropriate reference wavelet because it is well resolved from the direct waves traveling above and below the airwater interface.

I measured the spectral maxima along the first reflection event (permafrost table on the banks and water bottom in the pond) by using the STFT with a 20-ns moving window. With the frequency-shift method, I find that $1 / D$ for the pond water is $17 \pm 3$; the error is estimated as twice the standard deviation. The relationship for the temperature-dependent complex permittivity of water given by Olhoeft (1981) and equation 11 yields $Q^{*}=24$ at $200 \mathrm{MHz}$ and $0^{\circ} \mathrm{C}$. Given the complexities of measuring $Q^{*}$ from field data, the measured value of $1 / D$, although outside the error bounds, is quite close to $Q^{*}$. Deviation of the field measurement is likely caused by interference from underlying stratigraphic reflections, error in the referencespectrum measurement, and frequency-dependent reflection that re- sults from differing dispersion curves across the reflecting boundary. Perhaps the more significant result here is that despite a greater than $100 \%$ increase in traveltime and significant variability in the nearsubbottom reflectivity, $D$ remains approximately constant. This result indicates that it is possible to measure $D$ reliably in field data.

\section{Detection of NAPL contaminants}

Of many potential uses for GPR attenuation analysis, detection of nonpolar organic liquid contaminants may prove a particularly valuable application. These contaminants are referred to collectively as nonaqueous-phase liquids (NAPLs) because of their low solubility in water. NAPLs are subcategorized by their density relative to water; dense NAPLs (DNAPLs) are denser than water, and light NAPLs (LNAPLs) are lighter than water. It follows that DNAPLs tend to sink through the water column until they reach a low-permeability layer, whereas LNAPLs tend to remain near the water table with free product floating on the water column and residual product present in a smear zone above and below the water table. Both LNAPLs and DNAPLs leave a zone of residual contamination along their migration routes. Chlorinated solvents (DNAPL) and fuel hydrocarbons (LNAPL) are common examples.

NAPLs typically have low relative permittivity $\left(\varepsilon / \varepsilon_{0} \approx 2.5\right)$ and low electric conductivity ( $\sigma \approx 0.01-0.1 \mathrm{mS} / \mathrm{m}$ ) relative to freshwa$\operatorname{ter}\left(\varepsilon / \varepsilon_{0} \approx 81, \sigma>1 \mathrm{mS} / \mathrm{m}\right)$, which make them attractive targets for characterization using electrical geophysical methods. Of particular
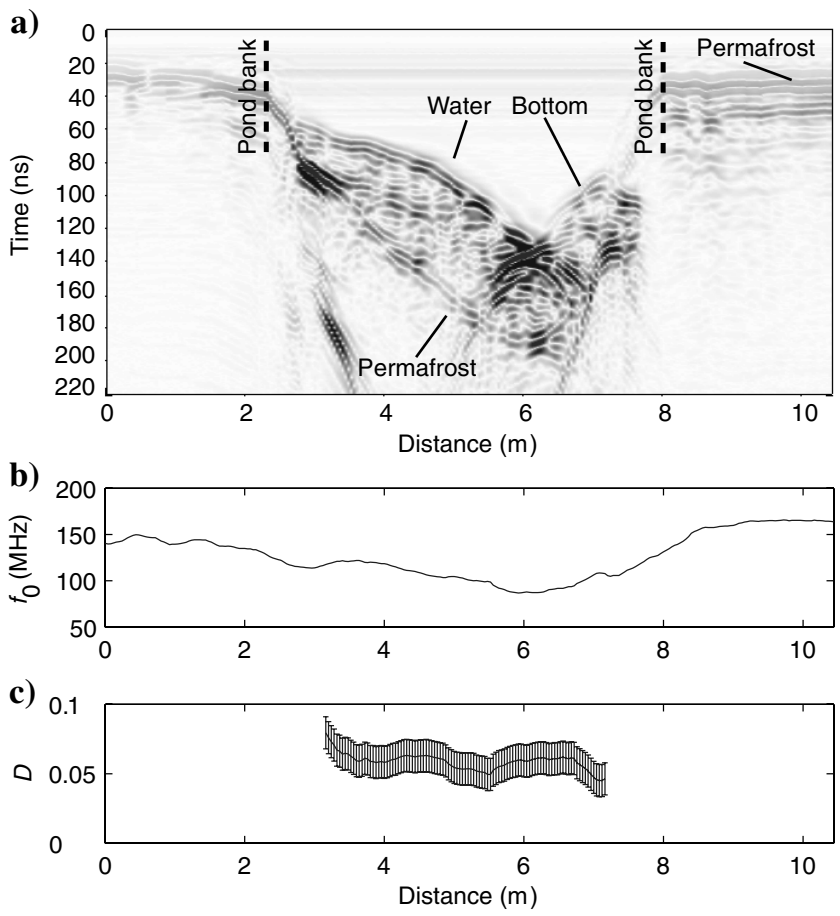

Figure 6. Data acquired over a pond on Alaska's North Slope. (a) GPR data at $200 \mathrm{MHz}$. (b) Maxima of spectral amplitudes $f_{0}$ of the first reflection, which is the top-of-permafrost on the banks and the water bottom within the pond. The conductivity of the pond water was roughly $45 \mu \mathrm{S} / \mathrm{cm}$, and the temperature was about $0^{\circ} \mathrm{C}$. (c) The dimensionless dispersion parameter $D$ for the pond water measured by using the frequency-shift method and the permafrost reflection between 9 and $10 \mathrm{~m}$ for the reference time and frequency. The $2 \sigma$ confidence bound is shown. $D$ is approximately constant and independent of traveltime as expected. 
importance for this study, NAPLs are electrically nondispersive. As the NAPL displaces water in the sediment pore space, a zone of anomalous electric properties may be induced. A first-order conceptual model is based on the premise that NAPL-contaminated zones will have low permittivity and low conductivity relative to the surrounding formation. A number of controlled-spill experiments have been consistent with this model (DeRyck et al., 1993; Brewster and Annan, 1994; Campbell et al., 1995; Bradford, 2004). This model has also proved effective in the interpretation of geophysical data acquired over a fresh LNAPL spill (Orlando, 2002) and over aged LNAPL and DNAPL spills at another field site (Lien and Enfield, 1998; Newmark et al., 1998; Orlando, 2002; Bradford and Deeds, 2006).

However, aged LNAPL spills at some sites have anomalously high electric conductivity (Monier-Williams, 1995; Sauck, 1998; Sauck et al., 1998; Atekwana et al., 2000; Bradford and Deeds, 2006). This divergence stems from the incorrect assumption that the electric properties of the unaltered LNAPL control the electric properties of the contaminated zone. Sauck (1998) proposes a conceptual model that explains the high-conductivity LNAPL plume in terms of increased dissolved-solid concentrations in the groundwater related to aerobic biogenic degradation of the hydrocarbons. Conversely, the mechanism for DNAPL degradation is anaerobic, and I am unaware of any DNAPL plumes reported to have high conductivities caused by weathering of the contaminant. In either case, frequencydependent GPR attenuation analysis only requires that an anomaly in the dispersion characteristics of the material be present.

Inext discuss two field sites for which qualitative analysis of the $D$ parameter is a strong indicator of lateral changes in relaxation characteristics related to the presence of NAPL. In both cases, I compute $D$ in the poststack domain from NMO-corrected, stacked multioffset GPR data. Computing $D$ from poststack data means that, in addition to averaging over a range of travel paths, the resulting measurement includes the effects of NMO stretch along with errors in moveout velocity. However, stacking also improves the S/N. I am not claiming to make a quantitative estimate of the intrinsic attenuation, which would require prestack analysis and a priori knowledge of the subsurface. Rather, I am looking for relative variability in the response, and the poststack approach is reasonable as long as lateral velocity gradients are small.

\section{Cape Fear wood-preserving site (creosote)}

The Cape Fear wood-preserving site is located in Fayetteville, North Carolina. The facility produced creosote-treated wood between 1953 and 1978. In 1977, the site was found to be contaminated with coal tar and coal tar creosote, the primary constituents of which are polyaromatic hydrocarbons (PAHs), a common DNAPL. In the mid-1980s, some remediation activities were initiated; these included excavation and removal of some contaminated soils. In 2001 and 2002, additional characterization revealed that significant quantities of residual and free-phase DNAPL remained. Current activities include a new round of characterization and remediation design. Shallow sediments at the site consist of fine-grained sands and silty sands to a depth of about $10 \mathrm{~m}$ where a thick silty clay unit is present. A shallow (1-m) water table is present in the unconfined surficial aquifer (Black and Veach, 2002).

The primary objectives of the radar study at the Cape Fear site were (1) to map the shallow stratigraphy and (2) to test methods for direct detection of DNAPL. Initial tests indicated that the site was conducive to radar investigation, with good data quality to depths of 6-11 m.

Line 2 (Figure 7) is typical of data acquired elsewhere at the site. The profile was placed to span from an area of zero or low contamination (on the left) to a zone of high contamination. To broaden the signal spectrum, I acquired coincident $50-$ and $100-\mathrm{MHz}$ profiles by using a Sensors and Software PE 100A system. The data were acquired in continuous common-midpoint (CMP) mode with 25-fold common-source gathers, 0.6-m source spacing, and 0.3-m receiver spacing. Prior to attenuation analysis, the processing stream consisted of a time-zero drift correction, high-pass dewow filter, $t^{2}$ gain correction, stacking velocity analysis, NMO correction, and stacking. Stacking the 100- and 50-MHz records served to broaden the source spectrum and to increase the $\mathrm{S} / \mathrm{N}$, thereby improving the precision of the analysis. The resulting spectrum (Figure $7 \mathrm{~b}$ ) was dominated by the 50-MHz signal because lower-frequency antennas radiate more power into the ground.

Laterally coherent reflections were recorded at depths as great as $11 \mathrm{~m}(\sim 250 \mathrm{~ns})$ (Figure 7a). For the attenuation analysis, I picked three horizons bounding two depth intervals: 3-7 m (70-190 ns) and 7-9 $\mathrm{m}$ (190-250 ns). These two intervals are bounded by relatively high-amplitude reflections and thus represent significant stratigraphic units. Note that from 70 to $82 \mathrm{~m}$ along line 2, the interval from the surface to a depth of $6 \mathrm{~m}(\sim 125 \mathrm{~ns})$ previously was excavated and backfilled and therefore is not representative of the natural stratigraphy.

I computed the time-frequency transform by using the STFT method and then measured the spectral maximum at every trace for the three horizons (Figure $7 b) . D$ was computed at each horizon relative to a laterally variable reference frequency (Figure $7 \mathrm{c}$ and d). The reference frequency was set by scaling the spectral peak along the shallowest horizon and had a mean of $68 \mathrm{MHz}$. The plotted value of $D$ is a measure of the cumulative frequency-dependent attenuation effects from the surface to each horizon. The dispersion image is computed by linear interpolation of $D$ along the vertical axis.

A significant low in the $D$ distribution thickens and deepens toward the west (Figure 7). This zone correlates very well with the spatial distribution of DNAPL (Figure 7d) measured by using conepenetrometer (CPT) mounted, laser-induced fluorescence (LIF) probes and direct borehole samples measured along an adjacent transect about $15 \mathrm{~m}$ to the south of line 2 (Black and Veach, 2002). The presence of DNAPL in the subsurface at this site produces a significant decrease in the dispersive properties of the bulk subsurface materials, and GPR is sensitive to this change.

\section{Former Wurtsmith Air Force Base (light hydrocarbons)}

This site is a former fire training facility, designated FT-02, located on the now decommissioned Wurtsmith Air Force Base in Oscoda, Michigan. Over a period of about 24 years, large quantities of fuel were burned on open ground during weekly training exercises. A significant volume of hydrocarbons did not burn; instead, they seeped into the underlying aquifer. In 1982, a concrete catch basin was constructed to minimize the amount of contaminant reaching the subsurface. By the early 1990s, the free-product plume was as thick as $0.3 \mathrm{~m}$ and extended more than $180 \mathrm{~m}$ down gradient from FT-02 (Bermejo et al., 1997; Sauck et al., 1998).

The stratigraphy below the site consists of fine- to mediumgrained sand and gravel deposits extending to a depth of approxi- 
mately $20 \mathrm{~m}$. Below these deposits is a silty clay layer 6-30 m thick that is thought to be the lower boundary for contaminant migration. The surficial aquifer is unconfined, with the water table present 3-5 m below the surface. The site was formerly a National Environmental Technology Test Site (NETTS) and was the site of a longterm natural bioremediation investigation. Although no longer an active NETTS, the wealth of characterization data available make this an excellent location for a semicontrolled GPR field experiment.

Central to the selection of this site for the current study are a series of geophysical investigations carried out by Sauck et al. (1998) and Bermejo et al. (1997). Their results point to excellent conditions for GPR because the site yields strong reflections well below the water table. Additionally, they cite a well-defined, high-attenuation anomaly coincident with the LNAPL plume. Through resistivity and self-potential measurements, and by inference from the GPR data, they conclude that high electric conductivity is associated with both the LNAPL and the dissolved phase plume. This site provided an excellent opportunity to study a conductive-type plume.

During July 2002, 1676 linear m of multioffset data were acquired, including a $930-\mathrm{m}^{2} 3 \mathrm{D}$ survey in both transverse electric (TE) and transverse magnetic (TM) modes. The 3D survey was oriented such that one edge of the plume would cross diagonally through the $3 \mathrm{D}$ patch. Additionally, data were acquired along two 122-m-long east-west profiles that bounded the 3D patch to the north and south. These profiles extended well beyond the east and west boundaries of the plume.

Along one of the $122-\mathrm{m}$ profiles, data were acquired with $100-\mathrm{MHz}$ antennas in continuous CMP mode with 25-fold common-source gathers, 0.6-m source interval, and a $0.3-\mathrm{m}$ receiver interval. The processing stream for attenuation analysis was the same as that described for the Cape Fear site, with the exception that a constant reference frequency of $90 \mathrm{MHz}$ was used. This reference frequency was the average spectral maximum of the direct arrival through the ground at an offset of $2.2 \mathrm{~m}$, where the arrival was well separated from the airwave.

The first objective was to reproduce the results of Sauck et al. (1998), and I found a similar attenuation anomaly. The zone of increased conductivity is most evident as an area of low reflection amplitudes, or shadow zone, beginning just below the water table and extending to depth (Figure $8 \mathrm{a})$. For the attenuation analysis, I interpreted two horizons: the water table reflection at approximately $65 \mathrm{~ns}(\sim 4 \mathrm{~m})$ and a horizon that varied from 120 to $150 \mathrm{~ns}$ (2-3 m below the water table) within the water-saturated zone. The deeper horizon is laterally discontinuous from a lateral position of $0-25 \mathrm{~m}$, then continuous across the rest of the profile. The measured $D$ distribution shows a distinct zone of increased dispersion that correlates with the zone of increased DC conduc-

d) tivity (Figure 8c). It is likely that the increase in DC conductivity in the pore fluid alters the relaxation characteristics of the bulk formation and masks any decrease in dispersion caused by the residual LNAPL that remains in the system.

Of additional interest at this site is that the $D$ value is generally higher in the vadose zone than in the saturated zone. This observation could indicate increased dispersion in the unsaturated sediments, as in the synthetic model in Figure 1, or could be caused by a decrease in the frequency content of the water table reflection because of a diffuse boundary at the capillary fringe.

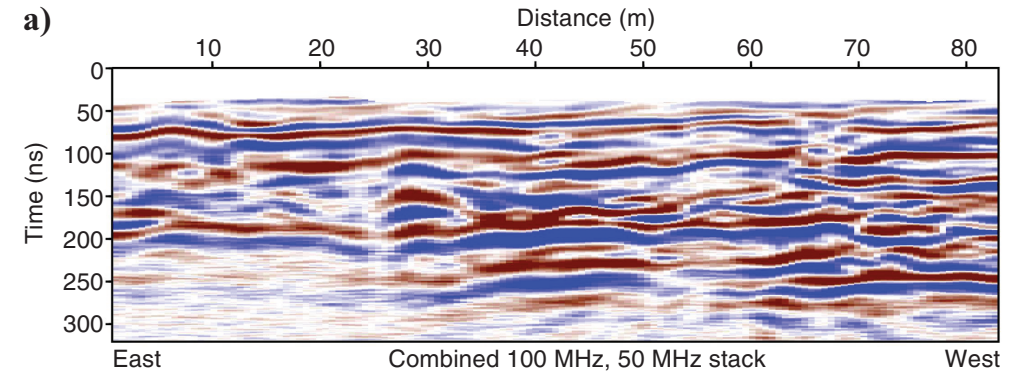

b) Peak frequency distribution
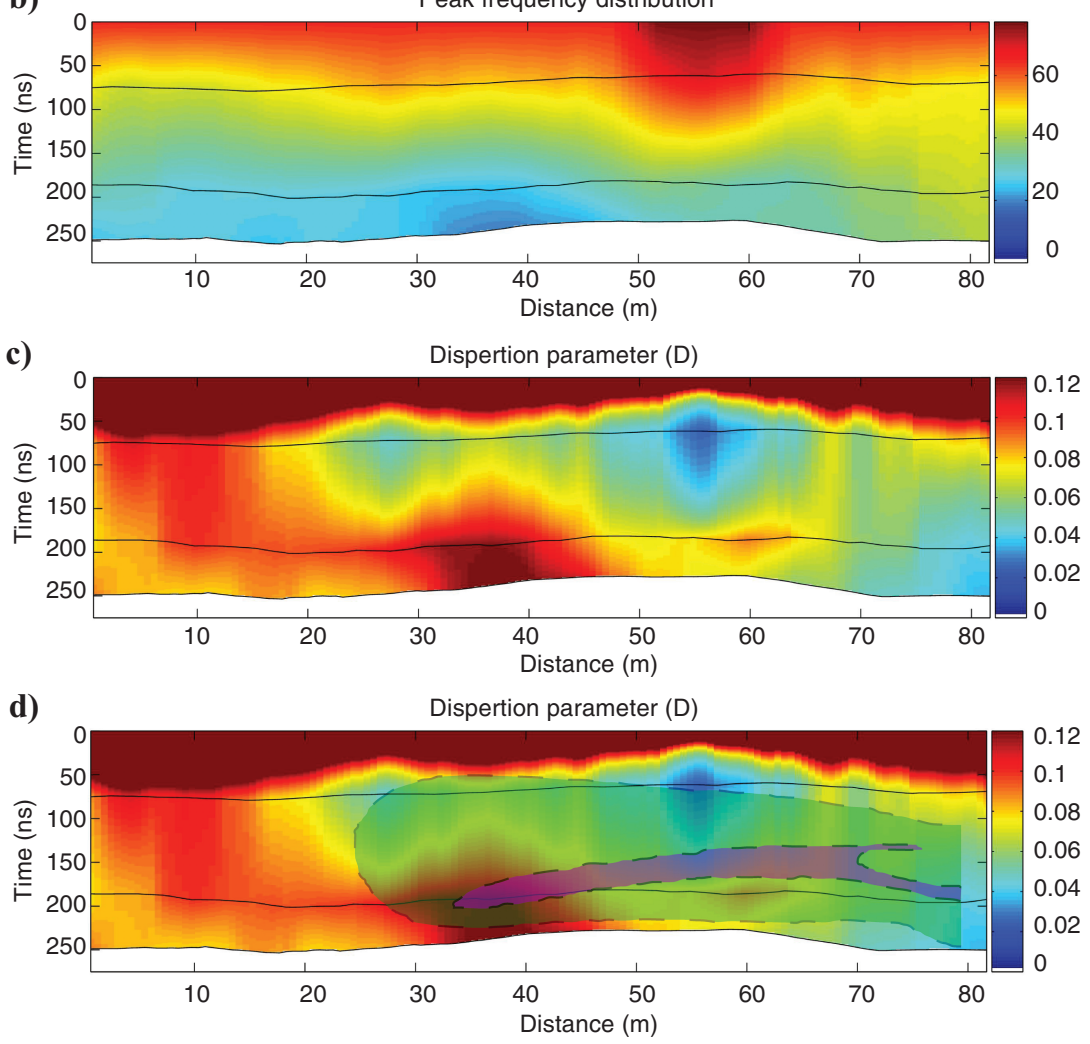

Figure 7. Data from the Cape Fear site. (a) Combined 50- and 100-MHz 25-fold stack. The soil disturbance caused by excavation and backfill is evident from 70 to $82 \mathrm{~m}$. (b) The peak-frequency distribution. (c) The $D$ distribution shows a zone of decreased dispersion that increases in thickness toward the west. (d) The $D$ distribution of significant creosote accumulations mapped by using CPT LIF and borehole-sampling logs shown in the shaded area. The DNAPL zone was projected from logs located approximately $50 \mathrm{ft}$ north of the profile. The green area (the elongated oval) shows elevated DNAPL concentration, and the purple area (the highly elongated, curved strip) shows high DNAPL concentration. The GPR horizons used in the analysis are indicated with black lines. 

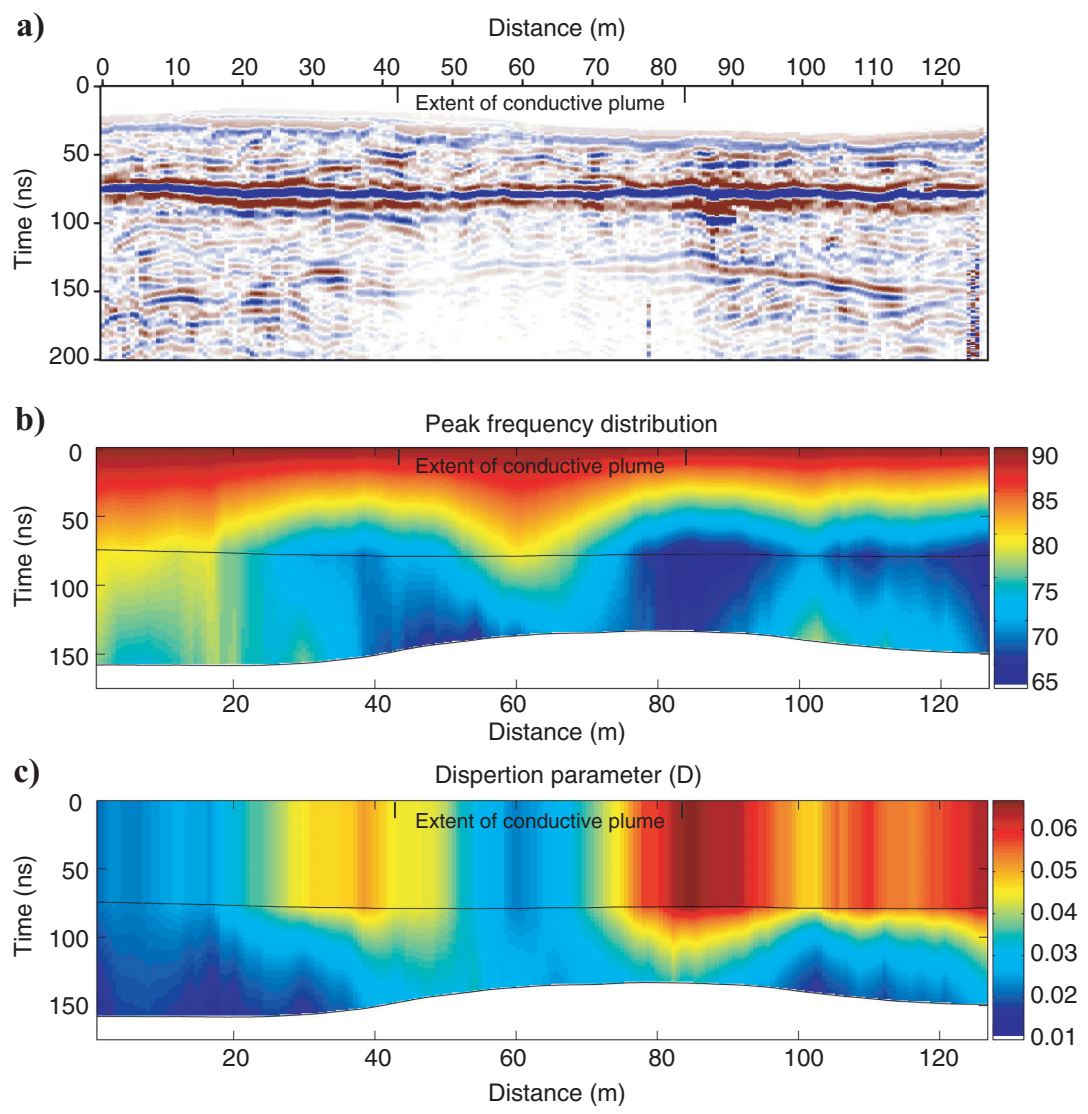

Figure 8. Data acquired at the former Wurtsmith Air Force Base. (a) CMP25-fold stack of $100-\mathrm{MHz}$ data. The water table reflection is at about $65 \mathrm{~ns}$. (b) The peak frequency distribution. (c) Image of the dispersion parameter $D$. At this site, increased dispersion below the water table correlates with increased DC conductivity caused by higher concentrations of dissolved solids in the groundwater. The $D$ anomaly indicates that the increase in DC conductivity of the fluid altered the relaxation mechanism of the bulk system. This increase in dissolved solids is a by-product of biodegradation of the hydrocarbon plume (Sauck, 1998; Sauck et al., 1998). GPR horizons used in the analysis are indicated with black lines.

\section{CONCLUSIONS}

Linearizing the imaginary component of the wavenumber in frequency by using the low-loss approximation provides a general formulation for the band-limited, constant- $Q^{*}$ model. Then $Q^{*}$ is related easily to any differentiable form for the complex dielectric permittivity, which may simplify the modeling problem by reducing the number of necessary material parameters as in the case for the ColeCole model. It is important to recognize that scattering and frequency-dependent reflection may alter the spectrum in reflection data and in some cases may be the predominant mode of spectral distortion. For this reason, I define the dispersion parameter $D$, whose measurement is mathematically equivalent to $1 / Q^{*}$, but the new parameter definition recognizes that the intrinsic attenuation is only one contributor to spectral shifts observed in GPR data. Significant contributors to the dispersion parameter are the intrinsic frequency-dependent electric properties at high frequencies and changes in those properties across reflecting boundaries. Although potentially correlated, $D$ provides fundamentally different information than low-frequency EM measurements.

At two field sites, I have shown that $D$ is a qualitative indicator of dielectric relaxation anomalies caused by the presence of organic contaminants. At the Cape Fear creosote site, the presence of DNAPL produced a zone of low dispersion, whereas at the former Wurtsmith Air Force Base, increased conductivity caused by biodegradation of LNAPL contamination produced a zone of relatively high dispersion.

\section{ACKNOWLEDGMENTS}

The U. S. Department of Energy (grant DEFG07-99ER15008) and U. S. Environmental Protection Agency (grant X-97008501-0) contributed funding for this research. Successful completion of this work would not have been possible without field assistants Allen Tanner, Mark Nell, and Alex McKinley. Boise State University acknowledges support of this research by Landmark Graphics Corporation via the Landmark University Grant Program.

\section{APPENDIX A}

\section{PERMITTIVITY DERIVATIVES FOR COLE-COLE RELAXATION}

Given the Cole-Cole relationship for the complex permittivity, I separate the real and imaginary components to give the following relationships:

$$
\varepsilon^{\prime}=\varepsilon_{\infty}+\frac{\left(\varepsilon_{\mathrm{DC}}-\varepsilon_{\infty}\right)\left[1+(\omega \tau)^{\gamma} \cos \frac{\pi \gamma}{2}\right]}{1+2(\omega \tau)^{\gamma} \cos \frac{\pi \gamma}{2}+(\omega \tau)^{2 \gamma}}
$$

and

$$
\varepsilon^{\prime \prime}=\frac{\left(\varepsilon_{\mathrm{DC}}-\varepsilon_{\infty}\right)(\omega \tau)^{\gamma} \sin \frac{\pi \gamma}{2}}{1+2(\omega \tau)^{\gamma} \cos \frac{\pi \gamma}{2}+(\omega \tau)^{2 \gamma}} .
$$

Taking the derivatives, I find

$$
\begin{aligned}
\frac{\delta \varepsilon^{\prime}}{\delta \omega}= & \frac{\varepsilon^{\prime \prime}}{\omega^{\gamma+1} \tau^{\gamma} \sin \frac{\pi \gamma}{2}}\left[\gamma(\omega \tau)^{\gamma} \cos \frac{\pi \gamma}{2}\right. \\
& \left.-\frac{2 \gamma\left[1+(\omega \tau)^{\gamma} \cos \frac{\pi \gamma}{2}\right]\left[(\omega \tau)^{\gamma} \cos \frac{\pi \gamma}{2}+(\omega \tau)^{2 \gamma}\right]}{1+2(\omega \tau)^{\gamma} \cos \frac{\pi \gamma}{2}+(\omega \tau)^{2 \gamma}}\right]
\end{aligned}
$$

and 


$$
\frac{\delta \varepsilon^{\prime \prime}}{\delta \omega}=\frac{\varepsilon^{\prime \prime}}{\omega}\left[1-\frac{2\left[(\omega \tau)^{\gamma} \cos \frac{\pi \gamma}{2}+(\omega \tau)^{2 \gamma}\right]}{1+2(\omega \tau)^{\gamma} \cos \frac{\pi \gamma}{2}+(\omega \tau)^{2 \gamma}}\right] .
$$

Note that equations 11, A-3, and A-4 differ from those originally given by Bradford (2006) in several important ways. First, the set of equations given here does not assume $\delta \varepsilon^{\prime} / \delta \omega=0$. This correction is often small but can be significant, particularly near the relaxation frequency. Second, the original set of equations assumed that $\gamma$ is close to one. Although these may be fair approximations in many cases, the set of equations given in this paper is valid for any parameter set.

It is interesting to note that when $\left(\omega_{0} \tau\right) \gamma \gg 1$, equation 11 reduces to

$$
\frac{1}{Q^{*}}=\frac{\varepsilon_{\omega_{0}}^{\prime \prime}}{\varepsilon_{\omega_{0}}^{\prime}}(1-\gamma) .
$$

When $\left(\omega_{0} \tau\right) \gamma \ll 1$ (e.g., water below $\left.1 \mathrm{GHz}\right)$, equation 11 becomes

$$
\frac{1}{Q^{*}}=\frac{\varepsilon_{\omega_{0}}^{\prime \prime}}{\varepsilon_{\omega_{0}}^{\prime}}(1+\gamma) .
$$

Equation A-6 requires that the low-loss approximation holds at low frequencies, which must be tested on a case-by-case basis with the binomial expansion that leads to equation 7. For Debye relaxation (Debye, 1945), $\gamma=1$, and at high frequencies, equation A-5 shows that $Q^{*}$ goes to infinity, implying that the attenuation is frequency independent. At low frequencies, equation A- 6 gives $Q^{*}$ as proportional to twice the ratio of real to imaginary parts of the complex dielectric permittivity (the phase tangent).

\section{REFERENCES}

Atekwana, E. A., W. A. Sauck, and D. D. Werkema, Jr., 2000, Investigations of geoelectrical signatures at a hydrocarbon contaminated site: Journal of Applied Geophysics, 44, 167-180.

Bano, M., 2004, Modelling of GPR waves for lossy media obeying a complex power law of frequency for dielectric permittivity: Geophysical Prospecting, 52, 11-26.

Bermejo, J. L., W. A. Sauck, and E. A. Atekwana, 1997, Geophysical discovery of a new LNAPL plume at the former Wurtsmith AFB, Oscoda, Michigan: Groundwater Monitoring and Remediation, 17, 131-137.

Black and Veach Special Projects Corp., 2002, Final NAPL investigation report: Cape Fear Wood Preserving Site, Fayetteville, Cumberland County, North Carolina: U. S. Environmental Protection Agency.

Bradford, J. H., 1999, Characterizing shallow aquifers with wave-propagation based geophysical techniques: Imaging and attribute analysis: Ph.D. dissertation, Rice University.

- 2004, 3D multi-offset, multi-polarization acquisition and processing of GPR data: A controlled DNAPL spill experiment: Proceedings of the Symposium on the Application of Geophysics to Environmental and Engineering Problems (SAGEEP 2004), Environmental and Engineering Geophysical Society, 514-527.

, 2006, Frequency dependent attenuation analysis of ground-penetrating radar data: Proceedings of the Symposium on the Application of Geophysics to Environmental and Engineering Problems (SAGEEP 2006), Environmental and Engineering Geophysical Society, 1532-1544.

Bradford, J. H., and J. C. Deeds, 2006, Ground-penetrating radar theory and application of thinbed offset dependent reflectivity: Geophysics, 71, no. 3, K47-K57

Bradford, J. H., J. P. McNamara, W. Bowden, and M. N. Gooseff, 2005, Measuring thaw depth beneath peat-lined arctic streams using ground-penetrating radar: Hydrological Processes, 19, 2689-2699.

Bradford, J. H., and Y. Wu, 2007, Instantaneous spectral analysis: Time-frequency mapping via wavelet matching with application to 3D GPR con- taminated site characterization: Proceedings of the Symposium on the Application of Geophysics to Environmental and Engineering Problems (SAGEEP 2007), Environmental and Engineering Geophysical Society.

Brewster, M. L., and A. P. Annan, 1994, Ground-penetrating radar monitoring of a controlled DNAPL release: $200 \mathrm{MHz}$ radar: Geophysics, 59, 1211-1221.

Cai, J., and G. A. McMechan, 1999, 2-D ray-based tomography for velocity, layer shape, and attenuation from GPR data: Geophysics, 64, 1579-1593.

Campbell, D. L., J. E. Lucious, K. J. Ellefson, and M. Deszcz-Pan, 1995, Monitoring of a controlled LNAPL spill using ground-penetrating radar: Proceedings of the Symposium on the Application of Geophysics to Environmental and Engineering Problems (SAGEEP'95), Environmental and Engineering Geophysical Society, 511-517.

Chakraborty, A., and D. Okaya, 1995, Frequency-time decomposition of seismic data using wavelet-based methods: Geophysics, 60, 1906-1916.

Chang, P. Y., D. Alumbaugh, J. Brainard, and L. Hall, 2002, Using crossborehole ground penetrating radar attenuation tomography for characterizing soil properties in the vadose zone during a two-stage infiltration test: 72nd Annual International Meeting, SEG, Expanded Abstracts, 1563 1566.

Cole, K. S., and R. S. Cole, 1941, Dispersion and absorption in dielectrics: I. Alternating current characteristics: Journal of Chemical Physics, 9 , 341-351.

Day-Lewis, F. D., J. M. Harris, and S. M. Gorelick, 2002, Time-lapse inversion of crosswell radar data: Geophysics, 67, 1740-1752.

Day-Lewis, F. D., J. W. Lane, J. M. Harris, and S. M. Gorelick, 2003, Timelapse imaging of saline-tracer transport in fractured rock using differenceattenuation radar tomography: Water Resources Research, 39, 1290.

Debye, P. J., 1945, Polar molecules: Dover Publ. Inc.

DeRyck, S. M., J. D. Redman, and A. P. Annan, 1993, Geophysical monitoring of a controlled kerosene spill: Symposium on the Application of Geophysics to Environmental and Engineering Problems (SAGEEP'93), Environmental and Engineering Geophysical Society, Proceedings, 5-19.

Goldstein, S. E., T. C. Johnson, M. D. Knoll, W. Barrash, and W. P. Clement, 2003, Borehole radar attenuation-difference tomography during the tracer/timelapse test at the Boise Hydrogeophysical Research Site: Symposium on the Application of Geophysics to Environmental and Engineering Problems (SAGEEP 2003), Environmental and Engineering Geophysical Society, 147-162.

Grandjean, G., J. C. Gourry, and A. Bitri, 2000, Evaluation of GPR techniques for civil-engineering applications: Study on a test site: Journal of Applied Geophysics, 45, 141-156.

Griffiths, D. J., 1989, Introduction to electrodynamics: Prentice-Hall, Inc.

Hoekstra, P., and A. Delaney, 1974, Dielectric properties of soils at UHF and microwave frequencies: Journal of Geophysical Research, 79, 1699 1708.

Irving, J. D., and R. J. Knight, 2003, Removal of wavelet dispersion from ground-penetrating radar data: Geophysics, 68, 960-970.

Jackson, D. J., 1999, Classical electrodynamics: John Wiley \& Sons, Inc.

Lane, J. W., F. D. Day-Lewis, J. M. Harris, F. P. Haeni, and S. M. Gorelick, 2000, Attenuation-difference radar tomography: Results of a multipleplane experiment at the, U. S. Geological Survey fractured-rock research site, Mirror Lake, New Hampshire: GPR 2000, Proceedings of the 8th International Conference on Ground Penetrating Radar, International Society for Optical Engineering, 666-675.

Li, X., and T. J. Ulrych, 1996, Multiscale attribute analysis and trace decomposition: 66th Annual International Meeting, SEG, Expanded Abstracts, $1634-1637$.

Lien, B. K., and C. G. Enfield, 1998, Delineation of subsurface hydrocarbon contaminant distribution using a direct push resistivity method: Journal of Environmental and Engineering Geophysics, 2, 173-179.

Liu, L., J. W. Lane, and Y. Quan, 1998, Radar attenuation tomography using the centroid frequency downshift method: Journal of Applied Geophysics, 40, 106-116.

Liu, L., and Y. Quan, 1997, GPR attenuation tomography for detecting DNAPLs: Proceedings of the Symposium on the Application of Geophysics to Environmental and Engineering Problems (SAGEEP'97), Environmental and Engineering Geophysical Society, 241-243.

Monier-Williams, M., 1995, Properties of light non-aqueous phase liquids and detection using commonly applied shallow sensing geophysical techniques: Proceedings of the Symposium on the Application of Geophysics to Environmental and Engineering Problems (SAGEEP '95), Environmental and Engineering Geophysical Society, 1-15.

Morlet, J., G. Arens, E. Fourgeau, and D. Giardi, 1982a, Wave propagation and sampling theory - Part I: Complex signal scattering in multilayered media: Geophysics, 47, 203-221.

- $1982 \mathrm{~b}$, Wave propagation and sampling theory — Part II: Sampling theory and complex waves: Geophysics, 47, 222-236.

Newmark, R. L., W. D. Daily, K. R. Kyle, and A. L. Ramirez, 1998, Monitoring DNAPL pumping using integrated geophysical techniques: Journal of Environmental and Engineering Geophysics, 3, 7-13.

Olhoeft, G. R., 1981, Electrical properties of rocks, in Y. S. Touloukian, W. R. 
Judd, and R. F. Roy, eds., Physical properties of rocks and minerals: McGraw-Hill Book, Co., 257-330.

Olhoeft, G. R., and D. E. Capron, 1993, Laboratory measurements of the radio frequency electrical and magnetic properties of soils from Yuma, Arizona: U. S. Geological Survey Open-File Report 93-07.

, 1994, Petrophysical causes of electromagnetic dispersion: Proceedings of the 5th International Conference on GPR, Waterloo Center for Groundwater Research, 145-152.

Orlando, L., 2002, Detection and analysis of LNAPL using the instantaneous amplitude and frequency of ground-penetrating radar data: Geophysical Prospecting, 50, 27-41.

Peterson, J. E. J., 2001, Preinversion corrections and analysis of radar tomographic data: Journal of Environmental and Engineering Geophysics, $\mathbf{6}$, $1-18$

Powers, M. H., and G. R. Olhoeft, 1996, Modeling the response of leaking, buried pipes: Proceedings of the Symposium on the Application of Geophysics to Environmental and Engineering Problems (SAGEEP '95), Environmental and Engineering Geophysical Society, 525-534.

Quan, Y., and J. M. Harris, 1997, Seismic attenuation tomography using the frequency shift method: Geophysics, 62, 895-905.

Roberts, R. L., and J. J. Daniels, 1992, 3-D data collection and analysis for high-resolution GPR studies: 62nd Annual International Meeting, SEG, Expanded Abstracts, 360-363.

Robertson, J. D., and H. H. Nagomi, 1984, Complex seismic trace analysis of thin beds: Geophysics, 49, 344-352.
Sauck, W. A., 1998, A conceptual model for the geoelectrical response of LNAPL plumes in granular sediments: Proceedings of the Symposium on the Application of Geophysics to Environmental and Engineering Problems (SAGEEP '98), Environmental and Engineering Geophysical Society, 805-817.

Sauck, W. A., E. A. Atekwana, and M. S. Nash, 1998, High conductivities associated with an LNAPL plume imaged by integrated geophysical techniques: Journal of Environmental and Engineering Geophysics, 2, 203-212.

Taherian, M. R., W. E. Kenyon, and K. A. Safinya, 1990, Measurement of dielectric response of water-saturated rocks: Geophysics, 55, 1530-1541.

Tobbock, T., P. Steeghs, G. G. Drijkoningen, and J. T. Fokkema, 1996, Decomposition of seismic signals via time-frequency representations: 66th Annual International Meeting, SEG, Expanded Abstracts, 1638-1641.

Turner, G., 1994, Subsurface radar propagation deconvolution: Geophysics, $\mathbf{5 9}, 215-223$

Turner, G., and A. F. Siggins, 1994, Constant $Q$ attenuation of subsurface radar pulses: Geophysics, 59, 1192-1200.

Zeng, X., G. A. McMechan, and T. Xu, 2000, Synthesis of amplitude versus offset variations in ground-penetrating radar data: Geophysics, $\mathbf{6 5}$, $113-125$.

Zhou, C., and L. Liu, 2001, Nonlinear inversion of borehole-radar tomography data to reconstruct velocity and attenuation distribution in earth materials: Journal of Applied Geophysics, 47, 299-308. 